\title{
Silver nanoparticles prepared in presence of ascorbic acid and gelatin, and their electrocatalytic application
}

\author{
FERESHTEH CHEKIN* and SOMAYEH GHASEMI \\ Department of Chemistry, Ayatollah Amoli Branch, Islamic Azad University, Amol 678, Iran
}

MS received 10 August 2013; revised 11 December 2013

\begin{abstract}
In this paper, we reported a simple and low-cost procedure to synthesize silver nanoparticles (AgNPs) by using ascorbic acid as reducing agent and gelatin as stabilizer. The synthesized AgNPs were characterized by various means such as transmission electron microscope (TEM), powder X-ray diffraction (XRD) and energy-dispersive X-ray (EDX). TEM observations and XRD analysis demonstrated that the size of AgNPs is about $20 \mathrm{~nm}$. Silver nanoparticles modified with carbon-paste electrode (AgNPs-CPE) displayed excellent electrochemical catalytic activities towards hydrogen peroxide $\left(\mathrm{H}_{2} \mathrm{O}_{2}\right)$. The reduction overpotential of $\mathrm{H}_{2} \mathrm{O}_{2}$ was decreased significantly compared with those obtained at the bare CPE. The sensor responded linearly to hydrogen peroxide $\left(\mathrm{H}_{2} \mathrm{O}_{2}\right)$ in the concentration of 10-350 $\mu \mathrm{M}$, with detection limit of $5.6 \mu \mathrm{M}$ at $3 \sigma$ using amperometry. The studied sensor exhibited good reproducibility and long-term stability.
\end{abstract}

Keywords. Silver nanoparticles; gelatin; ascorbic acid; hydrogen peroxide.

\section{Introduction}

Nanocatalysis has recently been a rapidly growing field which involves the use of nanoparticles as catalysts for a variety of organic and inorganic reactions (HernándezSantos et al 2002; Roucoux et al 2002; Widegren and Finke 2003). Ag nanoparticles show excellent catalytic and electrocatalytic activities (Jana et al 1999; Zhang et al 2005). For the past few years, various rapid chemical methods have been developed for the synthesis of silver nanoparticles (Shankar et al 2004; Panacek et al 2006). Reduction of $\mathrm{AgNO}_{3}$ by reductants such as citrate (Turkevich et al 1951; Lee and Meisel 1982; Pillai and Kamat 2004), ascorbic acid (Sondi et al 2003; Velikov et al 2003) or borohydride (Creighton et al 1979; Ahmadi et al 1996) is among the most used methods for the preparation of silver nanoparticles in aqueous solution. The morphology and size of the nanoparticles can be controlled by using a suitable polymerization agent such as gelatin in chemical methods (Qin et al 2010). Gelatin, which is the denaturation product of collagen, consists of one single chain of amino acids. A gelatin hydrogel is a three-dimensional hydrophilic polymer network, which can provide a desirable water-rich buffering environment because of its attractive properties of film-forming ability, biocompatibility, non-toxicity, high mechanical strength and low cost (Falk et al 2002; Zhang et al 2004, 2006; Yao et al 2005; Qian and Yang 2006; Zhang and Gorski 2011).

*Author for correspondence (fchekin@yahoo.com)
Detection of hydrogen peroxide $\left(\mathrm{H}_{2} \mathrm{O}_{2}\right)$ has become extremely important in recent years because of its wide and varied applications. These include uses in the textile, paper, cleaning product and food industries (Patterson et al 1984; Schliefer and Heidemann 1989). Up to now, many techniques including spectrometry, titrimetry, chemiluminescence and electrochemistry have been employed for determination of $\mathrm{H}_{2} \mathrm{O}_{2}$ (Willner and Katz 2000; Xiao et al 2003; Song et al 2006; Guo et al 2007; Bui et al 2010). Among them, electrochemical technique is a promising tool for the construction of simple and low-cost sensors due to their high sensitivity, good selectivity and ease of operation (Bui et al 2010). The presence of nanoparticles in electrochemical sensors can decrease the overpotentials of many analytes that occur at unmodified electrodes. Metal nanoparticles (NPs), such as PtNPs, AgNPs, AuNPs and PdNPs, have been proposed as electrocatalysts for sensing $\mathrm{H}_{2} \mathrm{O}_{2}$, owing to their excellent conductivity, extraordinary electrocatalytic property and larger specific surface area. Among these materials, silver nanoparticles (AgNPs) show excellent electrocatalytic activity for $\mathrm{H}_{2} \mathrm{O}_{2}$ (Lian et al 2009; Song et al 2009; Zhao et al 2009). Recent studies (Welch et al 2005; Wu et al 2006) indicate that the size distribution of AgNPs plays an important role in their electrocatalytic activity for $\mathrm{H}_{2} \mathrm{O}_{2}$.

In this paper, we report on the construction of AgNPs using ascorbic acid as the reducing agent stabilized by gelatin. It was found that the AgNPs can effectively catalyze the $\mathrm{H}_{2} \mathrm{O}_{2}$ reduction. The linear range is estimated to be from 10 to $350 \mu \mathrm{M}$ and the detection limit is estimated to be $5.6 \mu \mathrm{M}$, at a signal-to-noise ratio of 3 . 


\section{Experimental}

\subsection{Chemicals and apparatus}

Gelatin from bovine skin, ascorbic acid and silver nitrate were purchased from Aldrich. A stock solution of $\mathrm{H}_{2} \mathrm{O}_{2}$, freshly diluted from $30 \%$ solution, was from Aldrich (Germany). Electrochemical measurements were performed with an Autolab Potentiostat/Galvanostat (Metrohm, Autolab, Amsterdam, The Netherlands). The threeelectrode system consisting of an AgNPs-CPE as working electrode, $\mathrm{Ag}|\mathrm{AgCl}| \mathrm{KCl}_{3 \mathrm{M}}$ as the reference electrode and a platinum wire as an auxiliary electrode (Metrohm, The Netherlands) was used in all voltammetric experiments. TEM images were taken using LEO-Libra 120 transmission electron microscope. Hitachi S-3500N scanning electron microscope (SEM) with energy-dispersive X-ray (EDX) spectroscopy was used for surface image measurements and chemical characterization of a specimen. The crystallization and purity of the synthesized samples were characterized by X-ray diffraction.

\subsection{Preparation of Ag nanoparticles}

The precursor solution to synthesize AgNPs was prepared by dissolving $1.8 \mathrm{~g}$ gelatin in the $80 \mathrm{~mL}$ deionized water. The solution was stirred at $60{ }^{\circ} \mathrm{C}$ and $10 \mathrm{~mL}$ silver nitrate solution (1 M) and $10 \mathrm{~mL}$ ascorbic acid (2 M) were added to the above solution with continuous stirring. Then the container was moved to a water bath at $80^{\circ} \mathrm{C}$. The stirring was continued for $30 \mathrm{~min}$. The final product was calcined at $500{ }^{\circ} \mathrm{C}$ for $8 \mathrm{~h}$ to obtain silver nanoparticles. Also, this method for the synthesis of AgNPs was compared with the reported papers and are given in table 1 (Bar et al 2009; Zielinska et al 2009; Qin et al 2010; Bhatte et al 2012; Kim et al 2012; Raja et al 2012). As can be seen from table 1, the present method is comparable with the results reported in the literature.

\subsection{Fabrication of AgNPs-CPE}

A 1\% (w/w) AgNPs-spiked carbon powder was made by dissolving the given quantity of AgNPs in diethyl ether and hand mixed with 99 times of its weight of graphite powder in a mortar and pestle. The solvent was evaporated by stirring a mixture of 1\% AgNPs-spiked carbon powder and paraffin was blended by hand mixing, and the resulting paste was placed in the bottom of a glass tube (with internal radius $1.5 \mathrm{~mm}$ ). The electrical connection was implemented by a copper wire lead fitted into a glass tube. A carbon paste electrode without AgNPs was used as a blank to determine the background current.

\section{Results and discussion}

\subsection{Characterization}

The XRD pattern of the synthesized AgNPs is illustrated

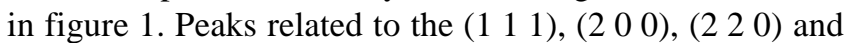
(3 11 1) diffractions of the face-central-cubic Ag structure (JCPDS no. 87-0597) can be seen, indicating that the Ag particles are made of pure crystalline Ag.

The TEM image (figure 2) of AgNPs is a useful approach to determine the size of Ag nanoparticles. The TEM image shows that the main particle size of the AgNPs, prepared with gelatin in presence of ascorbic acid, was about $20 \mathrm{~nm}$.

The EDX (figure 3) results confirmed the presence of respective constituent element in AgNPs.

\subsection{Electrochemical properties of AgNPs-CPE}

Cyclic voltammetry (CV) is one of the most common techniques used in electrochemistry to determine electroactivity. Figure 4 shows the cyclic voltammetric response obtained at the CPE and AgNPs-CPE in $0 \cdot 1 \mathrm{M}$ PBS (pH 7). At CPE (curve a), no obvious redox peak was observed, but a couple of quasi-reversible and welldefined peaks with $E_{1 / 2}$ of $390 \mathrm{mV}$ and $\Delta E$ of $220 \mathrm{mV}$ were observed at $20 \mathrm{mV} \mathrm{s}^{-1}$ for AgNPs-CPE (curve b) due to Ag nanoparticles.

The redox potential of the AgNPs-CPE is scan-rate dependent. Figure 5(A) shows the cyclic voltammograms of the AgNPs-CPE in 0.1 M PBS (pH 7) at various scan rates. It was observed that the values of $E_{\mathrm{pa}}$ and $E_{\mathrm{pc}}$ shift

Table 1. Comparable parameters for the synthesis of Ag nanoparticles by different methods.

\begin{tabular}{llccl}
\hline Synthesis procedure & Reducing agent & Stabilizer & Particle size (nm) & References \\
\hline Electron beam irradiation & Ag(I)-S12 polymer & Ag(I)-S12 polymer & $3 \cdot 1-100$ & Kim et al (2012) \\
Chemical & Hydrogen & Polyvinyl alcohol & 40 & Bhatte et al (2012) \\
Biosynthesis & Prosopis juliflora leaf & - & $35-60$ & Raja et al (2012) \\
Green synthesis & Jatropha curcas seed & - & $15-50$ & Bar et al (2009) \\
Wet chemical & Hydrazine & Polyvinyl alcohol & 48 & Zielinska et al (2009) \\
Wet chemical & Ascorbic acid & Polyvinyl alcohol & 44 & Zielinska et al (2009) \\
Wet chemical & Sodium borohydride & - & 140 & Zielinska et al (2009) \\
Green synthesis & Ascorbic acid & Citrate & $31-73$ & Qin et al (2010) \\
Green synthesis & Ascorbic acid & Gelatin & 20 & This work \\
\hline
\end{tabular}




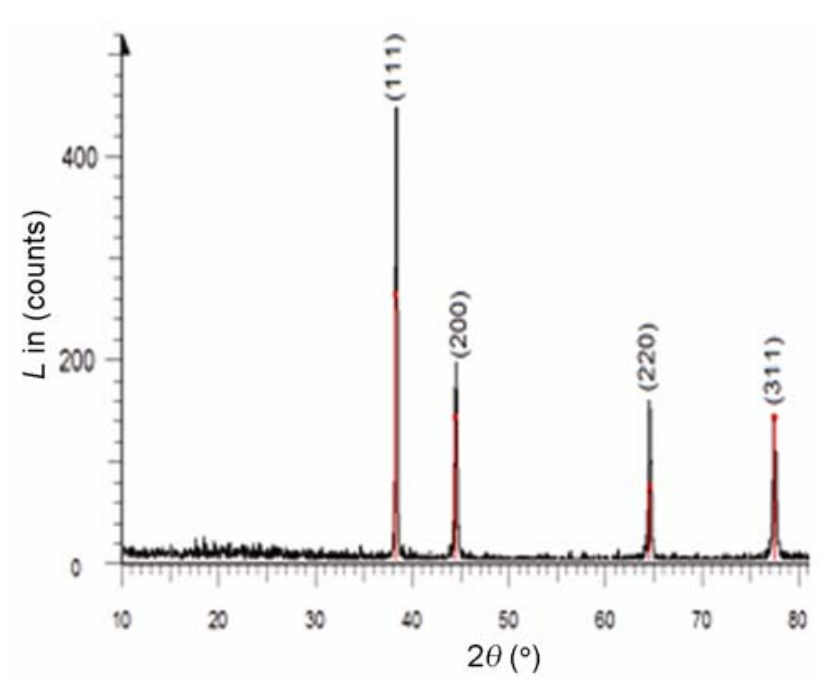

Figure 1. XRD pattern of the AgNPs.

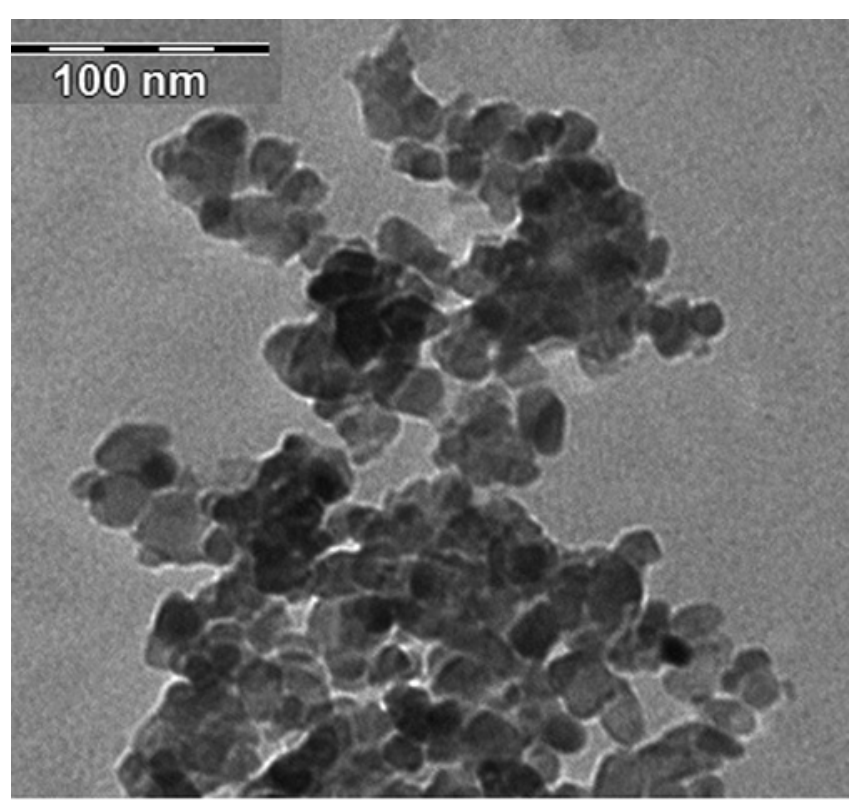

Figure 2. TEM image of AgNPs.

slightly to the positive and negative directions, respectively, and $\Delta E_{\mathrm{p}}$ increases with increasing scan rate. The anodic and cathodic peak currents are linearly proportional to scan rates (figure 5(B)), suggesting a surface-controlled process.

A plot of $E_{\mathrm{p}}$ vs $\log v$ yields a straight line with slopes of $-2 \cdot 3 R T / a n F$ and $2 \cdot 3 \mathrm{RT} /(1-a) n F$ for the cathodic and anodic peaks, respectively, so that $a$ can be estimated as 0.63 from the slope of the straight lines. The surface concentration of the electroactive Ag on AgNPs-CPE, $\Gamma$ (in $\mathrm{mol} / \mathrm{cm}^{2}$ ), can be estimated using the equation (Laviron 1979)

$$
\Gamma=Q / n F A,
$$

where $Q$ is the charge consumed in coulombs, obtained from integrating the anodic (or cathodic) peak area in

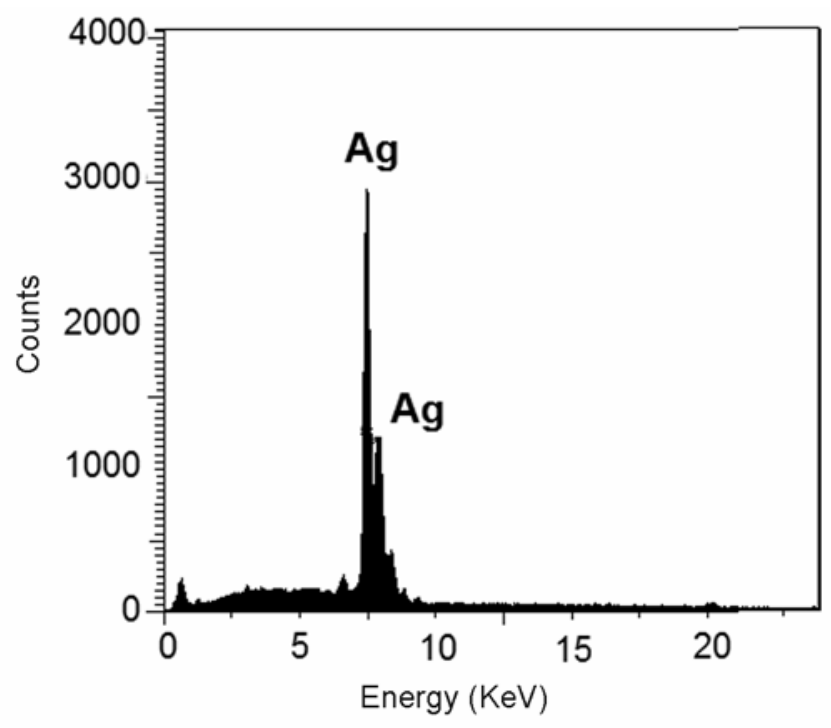

Figure 3. EDX spectrum of AgNPs.

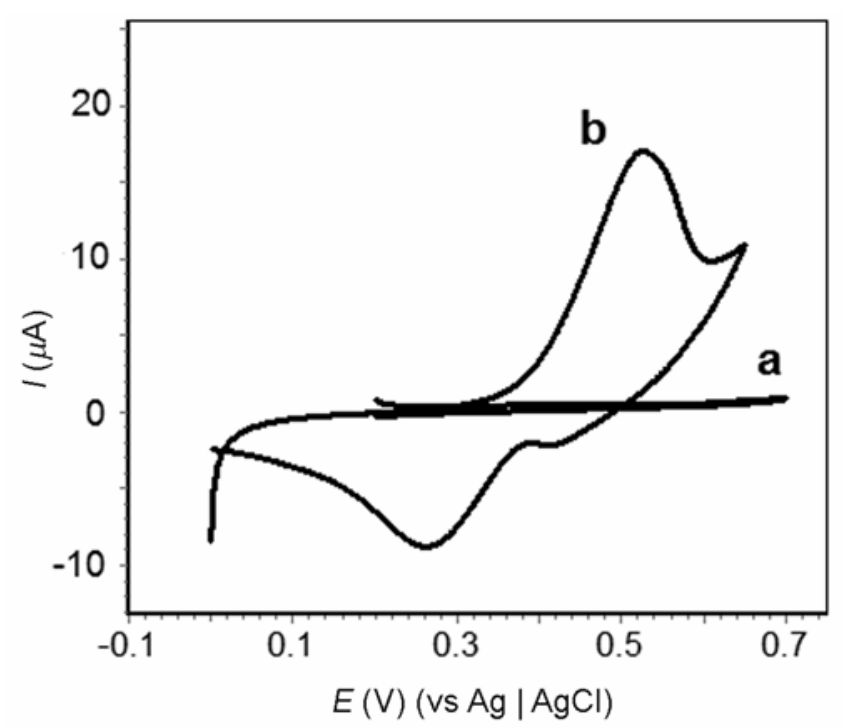

Figure 4. Cyclic voltammograms of the CPE (a) and AgNPsCPE (b) in PBS with pH 7 at a scan rate of $20 \mathrm{mV} \mathrm{s}^{-1}$.

cyclic voltammograms under the background correction. The average $\Gamma$ value of $(2 \cdot 73 \pm 0 \cdot 81) \times 10^{-10} \mathrm{~mol} / \mathrm{cm}^{2}$ was obtained.

\subsection{Electrocatalytic behaviour of AgNPs-CPE}

In order to investigate the electrocatalytic properties of the AgNPs-CPE, its response to the reduction of $\mathrm{H}_{2} \mathrm{O}_{2}$ was studied in a $\mathrm{pH} 7 \mathrm{PBS}$ using cyclic voltammetry. As shown in figure 6, at the AgNPs-CPE, the cathodic peak current at around $-150 \mathrm{mV}$ increases with increase of $\mathrm{H}_{2} \mathrm{O}_{2}$ concentration, demonstrating a typical electrocatalytic 

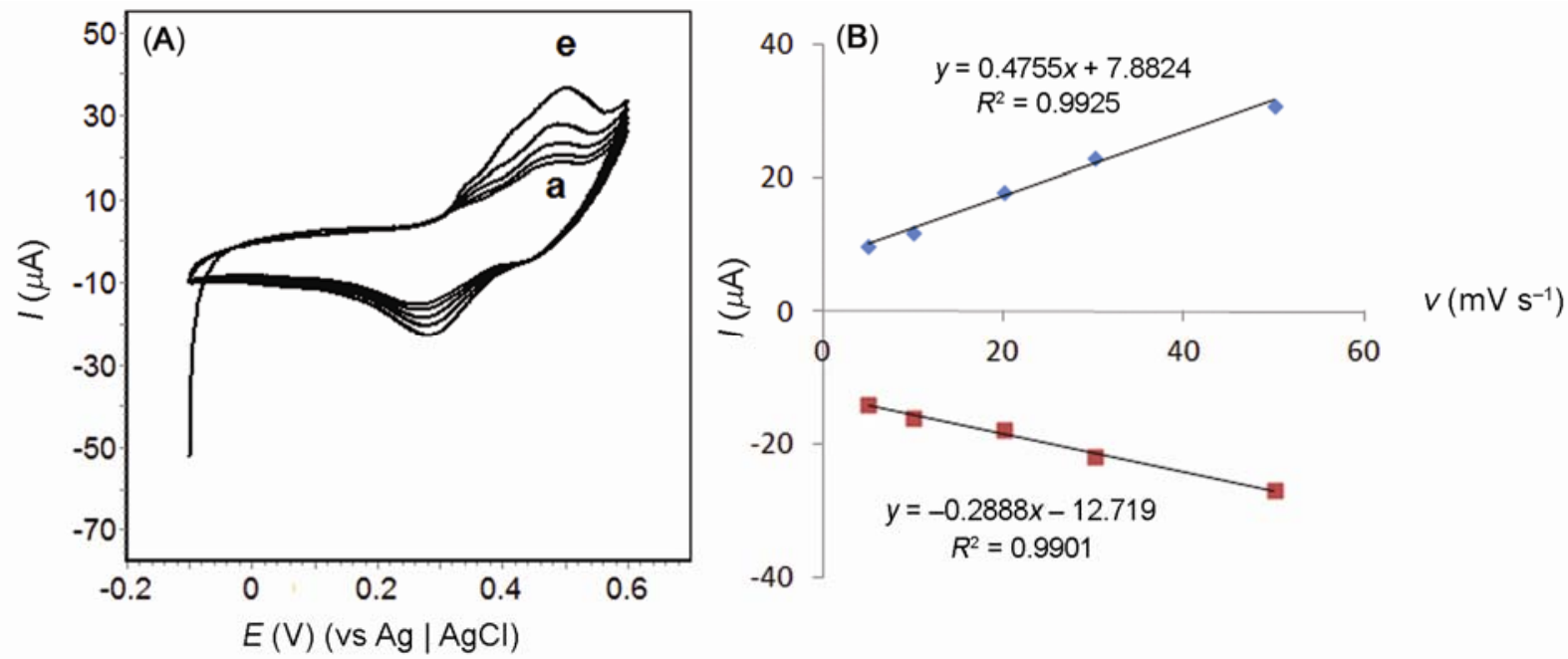

Figure 5. (A) Cyclic voltammograms of AgNPs-CPE in 0.1 M PBS solution (pH 7) at various scan rates: (a) 5, (b) 10 , (c) 20 , (d) 30 and (e) $50 \mathrm{mV} \mathrm{s}^{-1}$. (B) Plot of peak currents vs scan rates.

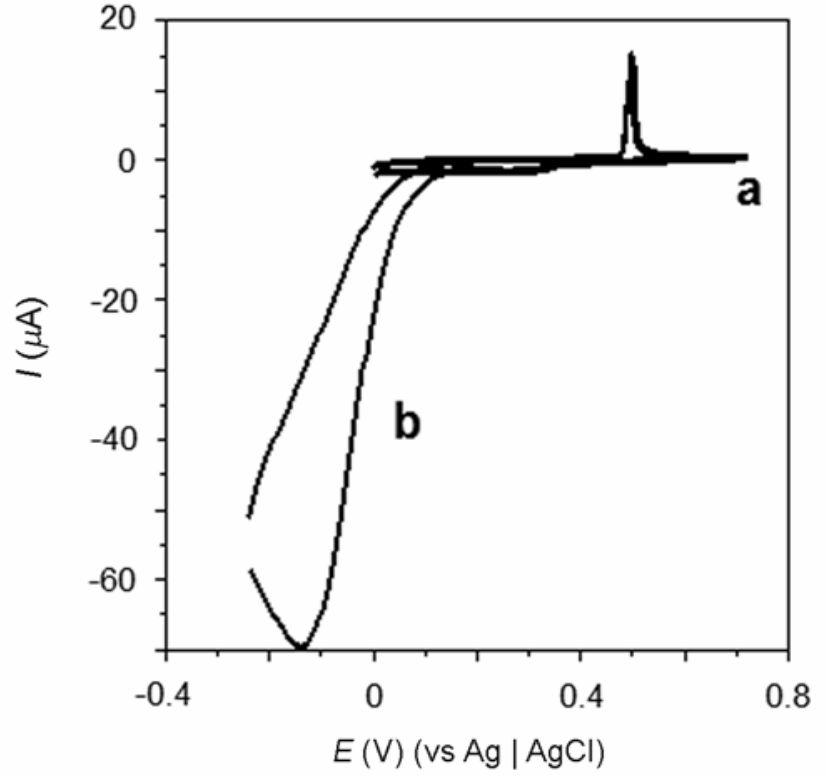

Figure 6. Cyclic voltammograms of AgNPs-CPE in the (a) absence and (b) presence of $1 \mathrm{mM}$ of $\mathrm{H}_{2} \mathrm{O}_{2}$ in $0 \cdot 1 \mathrm{M}$ PBS solution (pH 7) and $0 \cdot 1 \mathrm{M} \mathrm{KCl}$ as supporting electrolyte saturated with $\mathrm{N}_{2}$ at a scan rate of $25 \mathrm{mV} \mathrm{s}^{-1}$.

reduction process of $\mathrm{H}_{2} \mathrm{O}_{2}$. The effect of $\mathrm{pH}$ value on the reduction of $\mathrm{H}_{2} \mathrm{O}_{2}$ at the surface of AgNPs-CPE was investigated using different $0 \cdot 1 \mathrm{M}$ phosphate buffer solutions ( $\mathrm{pH}$ 3-9). The current response of $\mathrm{H}_{2} \mathrm{O}_{2}$ at AgNPs$\mathrm{CPE}$ increases from $\mathrm{pH} 3$ to 7 and then a decrease is observed at $\mathrm{pH}$ values higher than 7 (not shown). Therefore, $\mathrm{pH} 7$ was chosen as optimum $\mathrm{pH}$ and further studies were performed at $\mathrm{pH}=7$.

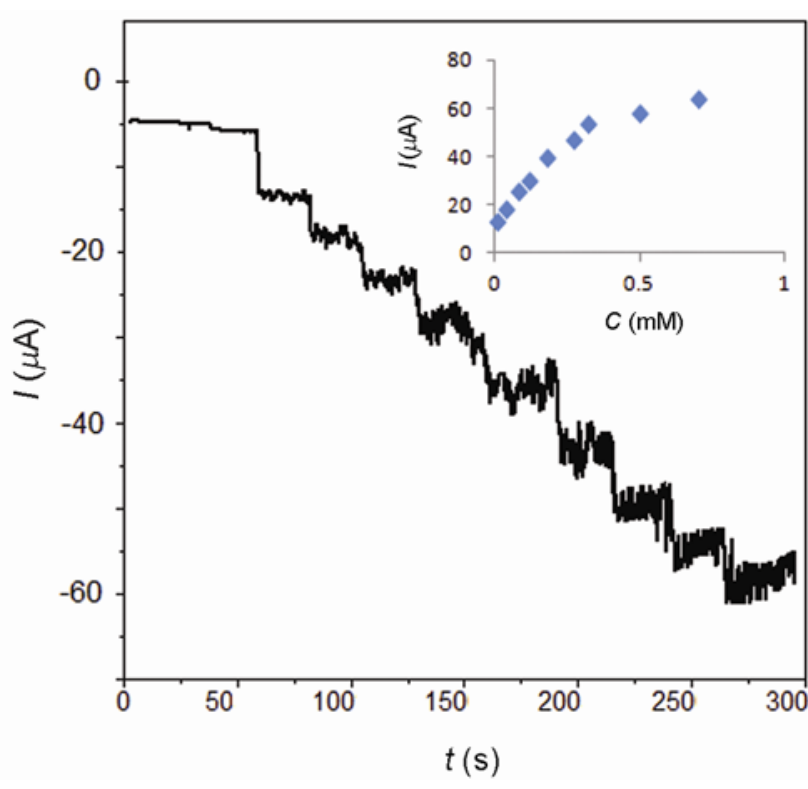

Figure 7. Current-time curve of AgNPs-CPE with successive addition of $\mathrm{H}_{2} \mathrm{O}_{2}$ to a stirred $0 \cdot 1 \mathrm{M}$ PBS (pH 7) and $0 \cdot 1 \mathrm{M} \mathrm{KCl}$ as supporting electrolyte saturated with $\mathrm{N}_{2}$. The inset is the calibration curve.

The $\mathrm{H}_{2} \mathrm{O}_{2}$ reduction on AgNPs-CPE was explored for amperometric responses of different concentrations of $\mathrm{H}_{2} \mathrm{O}_{2}$ at AgNPs-CPE with an applied potential of $-250 \mathrm{mV}$ in $0.1 \mathrm{M}$ PBS ( $\mathrm{pH} \mathrm{7)}$ (figure 7). We use the current value to plot with the concentration of $\mathrm{H}_{2} \mathrm{O}_{2}$ (inset). There was a linear relation of the current with concentration of $\mathrm{H}_{2} \mathrm{O}_{2}$ between 10 and $350 \mu \mathrm{M}$. Detection limit is $5.6 \mu \mathrm{M}$ of $\mathrm{H}_{2} \mathrm{O}_{2}$ with the signal-to-noise ratio of 3. Also, the sensor responded to $\mathrm{H}_{2} \mathrm{O}_{2}$ with sensitivity of $2533.8 \mu \mathrm{A} \mathrm{mM} \mathrm{cm}{ }^{-2}$. The analytical parameters are 
comparable with results reported for analytical determination of $\mathrm{H}_{2} \mathrm{O}_{2}$ at the surface of different modified electrodes (Song et al 2009a, b; Lin et al 2010; Chen et al 2011; Liu et al 2011; Lu et al 2011).

\subsection{Reproducibility and stability of the AgNPs-CPE}

The operational stability of AgNPs-CPE was tested by the $\mathrm{CV}$ method every 2 days in 2 weeks. The response to $1 \mathrm{mM} \mathrm{H}_{2} \mathrm{O}_{2}$ decreased less than $19 \%$ after 2 weeks, so it has good stability. The fabrication reproducibility of four electrodes independently made showed an R.S.D. of $8.9 \%$ for detecting $1 \mathrm{mM} \mathrm{H}_{2} \mathrm{O}_{2}$. The excellent reproducibility and stability of the sensor may be mainly attributed to the strong immobilization of the Ag nanoparticles in CPE.

\section{Conclusion}

We developed a novel green synthesis protocol of preparing silver nanoparticles (AgNPs) involving the reduction of silver nitrate in the presence of gelatin as stabilizer and ascorbic acid as reducing agent in aqueous medium. The prepared AgNPs were characterized by TEM, XRD and EDX and studied for their activity as a catalyst for the modification of CPE (AgNPs-CPE). This modified electrode exhibited high electrocatalytic activities towards the reduction of $\mathrm{H}_{2} \mathrm{O}_{2}$ by significantly decreasing their reduction overpotential and enhancing the peak current. This electrochemical sensor showed high sensitivity, good reproducibility and long-term stability.

\section{References}

Ahmadi T S, Wang Z L, Green T C, Henglein A and El-Sayed M A 1996 Science 2721924

Bar H, Bhui D K, Sahoo G P, Sarkar P, Pyne S and Misra A 2009 Coll. Surf. A: Physicochem. Eng. Aspects 348212

Bhatte K D, Deshmukh K M, Patil Y P, Sawant D N, Fujita Sh I, Arai M and Bhanage B M 2012 Particuology 10140

Bui M N, Pham X, Han K N, Li C A, Kim Y S and Seong G H 2010 Sen. Actuators B150 436

Chen H, Zhang Z, Cai D, Zhang S, Zhang B, Tang J and Wu Z 2011 Talanta 86266

Creighton J A, Blatchford C G and Albrecht M 1979 J. Chem. Soc. Faraday Trans. $\mathbf{7 5} 790$

Falk B, Garramone S and Shivkumar S 2002 Anal. Chem. 745039

Jana N R, Sau T K and Pal T 1999 J. Phys. Chem. B103 115

Guo C, Song Y, Wei H, Li P, Wang L, Sun L, Sun Y and Li Z 2007 Anal. Bioanal. Chem. 389527

Hernández-Santos D, González-García M B and García A C 2002 Electroanalysis 141225

Kim S E, Park J H, Lee B, Lee J Ch and Kwon Y K 2012 Radiat. Phys. Chem. 81978

Laviron E 1979 J. Electroanal. Chem. 100263
Lee P C and Meisel D 1982 J. Phys. Chem. 863391

Lian W, Wang L, Song Y, Yuan H, Zhao S, Li P and Chen L 2009 Electrochim. Acta 544334

Lin C Y, Lai Y H, Balamurugan A, Vittal R, Lin C W and Ho K C 2010 Talanta 82340

Liu S, Wang L, Tian J, Luo Y, Zhang X and Sun X 2011 J. Colloid Interf. Sci. 363615

Lu W, Luo Y, Chang G, Liao F and Sun X 2011 Thin Solid Films 520554

Panacek A, Kvitek L, Prucek R, Kolar M, Vecerova R and Pizurova N 2006 J. Phys. Chem. B110 16248

Patterson B D, MacRae E A and Ferguson I B 1984 Anal. Biochem. 139487

Pillai Z S and Kamat P V 2004 J. Phys. Chem. B108 945

Qian L and Yang X R 2006 Talanta 68721

Qin Y, Ji X, Jing J, Liu H, Wu H and Yang W 2010 Coll. Surf. A: Physicochem. Eng. Aspects 372172

Raja K, Saravanakumar A and Vijayakumar R 2012 Spectrochim. Acta A: Molec. and Biomolec. Spectrosc. 97490

Roucoux A, Schulz J and Patin H 2002 Chem. Rev. 1023757

Schliefer K and Heidemann G 1989 Investigation into the tendering mechanism in the peroxide bleaching of cotton caused by impurities containing catalysts (MCI) p 856

Shankar S S, Rai A, Ahmad A and Sastry M J 2004 J. Colloid Interf. Sci. 275496

Sondi I, Goia D V and Matijevic E 2003 J. Colloid Interf. Sci. 26075

Song Y, Wang L, Ren C, Zhu G and Li Z 2006 Sens. Actuators B114 1001

Song Y H, Cui K, Wang L and Chen S H 2009a Nanotechnology 20105501

Song Y, Cui K, Wang L and Chen S 2009b Nanotechnology 20 105501

Turkevich J, Stevenson P C and Hillier J 1951 Discuss. Faraday Soc. 1155

Velikov K P, Zegers G E and Blaaderen A V 2003 Langmuir 191384

Welch C, Banks C, Simm A and Compton R 2005 Anal. Bioanal. Chem. 38212

Widegren J A and Finke R G 2003 J. Mol. Catal. A: Chem. 191187

Willner I and Katz E 2000 Angew. Chem. Int. Ed. 391180

Wu S, Zhao H, Ju H, Shi C and Zhao J 2006 Electrochem. Commun. 81197

Xiao Y, Patolsky F, Katz E, Hainfeld J and Willner I 2003 Science 2991877

Yao T, Nanjyo Y, Tanaka T and Nishino H 2005 Electroanalysis 172114

Zhang Z J, Liu C Y and Sun L W 2005 J. Phys. Chem. B109 1730

Zhang M G, Smith A and Gorshi W 2004 Anal. Chem. 765045

Zhang M and Gorski W 2011 Electroanalysis 231856

Zhang M, Mullens C and Gorski W 2006 Electrochim. Acta 514528

Zhao B, Liu Z, Liu Z L, Liu G X, Li Z, Wang J X and Dong X 2009 Electrochem. Commun. 111707

Zielinska A, Skwarek E, Zaleska A, Gazda M and Hupka J 2009 Proc. Chem. 11560 